\title{
CALIDAD MICROBIOLÓGICA, FISICOQUÍMICA, DETERMINACIÓN DE NITRITOS Y TEXTURA DE CHORIZOS COMERCIALIZADOS EN CARTAGENA (COLOMBIA)
}

\section{MICROBIOLOGICAL QUALITY, PHYSICOCHEMICAL, DETERMINATION OF NITRITE AND TEXTURE OF SAUSAGES MARKETED IN CARTAGENA (COLOMBIA)}

\author{
Diego Tirado ${ }^{1}$, Diofanor Acevedo $^{2}$, Piedad Montero $^{3}$
}

\begin{abstract}
${ }^{1}$ Ingeniero de Alimentos. c.M.Sc. Ingeniería Ambiental. Universidad de Cartagena, Facultad de Ingeniería. Campus de Piedra de Bolívar, Cartagena-Bolívar, Colombia, e-mail: dtiradoa@unicartagena.edu.co; ${ }^{2}$ Ingeniero de Alimentos. Ph.D., Ingeniería de Alimentos. Universidad de Cartagena, Facultad de Ingeniería, Programa de Ingeniería de Alimentos, Avenida El Consulado, Calle 30 No, 48-152, Cartagena, Bolívar-Colombia, e-mail: diofanor3000@gmail.com; ${ }^{3}$ Ingeniera de Alimentos. M.Sc. Ciencia y Tecnología de Alimentos. Universidad de Cartagena, Facultad de Ingeniería, Programa de Ingeniería de Alimentos. Avenida El Consulado, Calle 30 No, 48-152, Cartagena, Bolívar-Colombia, e-mail: pmonteroc@unicartagena.edu.co
\end{abstract}

Rev. U.D.C.A Act. \& Div. Cient. 18(1): 189-195, Enero-Junio, 2015

\section{RESUMEN}

Muchos alimentos cárnicos procesados, como los chorizos, poseen elevados niveles de nitritos y algunos países han avanzado en el desarrollo de programas para reducir, significativamente, el contenido de estos en los alimentos procesados y el fomento de una disminución en su uso indiscriminado, ya que su consumo se asocia a enfermedades, como el cáncer, la diabetes, Parkinson y el Alzheimer. El presente estudio evaluó la calidad microbiológica, fisicoquímica, contenido de nitritos y textura en chorizos comercializados, en la ciudad de Cartagena (Colombia). El análisis proximal, se determinó mediante A.O.A.C. y la calidad microbiológica, según Norma Técnica Colombiana 1325. Se tomaron diez muestras al azar de chorizos de cerdo tipo coctel, comercialización en Supermercados, Mercado de Bazurto y venta callejera. El producto comercializado en supermercado presentó mayor contenido de proteína y menor de carbohidrato y almidón, que los obtenidos en las ventas callejeras y el mercado de Bazurto. Los comercializados en ventas callejeras y Mercado Bazurto revelaron valores de nitritos superiores a los permitidos por la Norma Técnica Colombiana. Los chorizos evaluados poseen un perfil de textura similar a los reportados por otros autores, para este tipo de productos. Los chorizos comercializados en supermercado son los únicos que cumplen con los requisitos microbiológicos expuestos en la Norma Técnica Colombiana, indicando mejor calidad nutricional, microbiológica y de textura.

Palabras clave: Calidad microbiológica, nitritos, textura, chorizo, calidad nutricional.

\section{SUMMARY}

Much meat processed foods like sausages have high levels of nitrites, and some countries have made progress in developing programs to significantly reduce the content of these in processed foods, and promoting a decrease in their indiscriminate use, as their consumption is associated with diseases such as cancer, diabetes, Parkinson's and Alzheimer's. This study evaluated the microbiological, physicochemical, nitrite content and texture in sausages sold in the city of Cartagena (Colombia) quality. Proximate analysis was determined by A.O.A.C. and microbiological quality according to Colombian Technical Standard 1325. Ten samples were taken at random from pork sausage cocktail type, marketing Supermarkets, Market Bazurto and street located in the city of Cartagena. The product sold in supermarket had a higher content of protein and carbohydrate and starch lower than those obtained in street sales and market Bazurto. The sold in street sales and market Bazurto values were higher than permitted by the Colombian Technical Standard nitrites. The sausages evaluated have a profile similar to those reported by other authors for this type of product texture. The sausages sold in supermarket are the ones that meet the microbiological requirements set out in the Colombian Technical Standard, presenting improved nutritional, microbiological and textural quality.

Key words: Microbiological quality, nitrites, texture, chorizo, nutritional quality. 


\section{INTRODUCCIÓN}

El chorizo es un embutido crudo, de origen español, que difiere muy poco de la longaniza, en cuanto a su composición (FAO, 2006). Se elabora a partir de carne picada de cerdo, mezclada con sal, especias y nitrato de potasio (FAO, 2006). El producto es embutido en tripa de cerdo y atado en fracciones de 10 a 25 centímetros (FAO, 2006). El chorizo, se destaca dentro de los embutidos más populares y extendidos en toda Latinoamérica (González-Tenorio et al. 2012; 2013). Su lugar de comercialización corresponde con el tipo de elaborador, ya sea en grandes industrias, que sustentan una marca registrada, cuyos chorizos se venden en supermercados; medianas empresas, de ámbito regional, que elaboran y distribuyen el chorizo en centrales de abasto mayoristas, carniceros locales minoristas o, pequeños productores de ganado, en el ámbito rural y familiar (González-Tenorio et al. 2013). A diferencia de los dos primeros grupos, los productores de los dos últimos elaboran los chorizos siguiendo formulaciones y procedimientos transmitidos de generación en generación, de forma tradicional (Guerrero et al. 2009). En su preparación, prácticamente, no se usan aditivos ni ingredientes no cárnicos distintos a la sal, las especias y los condimentos, lo que está en contraste con la tendencia actual de elaborar embutidos frescos de bajo costo, con la adición de proteínas no cárnicas y altos niveles de grasa, así como diversos aditivos: fosfatos, conservantes, saborizantes, colorantes, sustancias de relleno o azúcares (González-Tenorio et al. 2013).

En algunos países latinoamericanos, el chorizo se vende en forma cruda, requiriéndose una etapa de freído, antes de su consumo (FAO, 2006); no obstante, en el procedimiento tradicional el chorizo es desecado y ahumado, proceso en que la actividad acuosa se disminuye, hasta un punto de 0,6 a 0,75 , en que se impide el crecimiento microbiano (FAO, 2006). Durante el desecado ocurre la maduración del producto, que es un fenómeno bioquímico y microbiano muy complejo, donde se presentan tres fases importantes: el enrojecimiento, el aumento de consistencia y la aromatización (FAO, 2006).

La calidad en productos cárnicos procesados, como el chorizo, es un tema de interés en la industria alimentaria, por lo que ya se han realizado investigaciones en el tema (Wang et al. 2013). González \& Diez (2002) analizaron el efecto de los nitritos y cultivo iniciador en la calidad microbiológica de chorizo. Ambrosiadis et al. (2004) realizaron estudios fisicoquímicos, microbiológicos y sensoriales a 67 muestras de embutidos tradicionales griegos, mientras que Fernández-López et al. (2008) investigaron perfiles fisicoquímicos y microbiológicos de salchichones, enriquecidos con fibra de naranja. En general, estos estudios concluyen que las características de los productos cárnicos dependen, prin- cipalmente, del origen de los mismos, presentando claras diferencias en sus propiedades.

La venta de alimentos generada por vendedores ambulantes satisface la necesidad básica de comida de personas que se encuentran fuera del hogar o del sitio de trabajo, sin darse cuenta que los productos pueden ser una fuente de infección, debido a su inadecuada manipulación (Bayona, 2012). Según Bayona (2012) existe una relación directa entre la inadecuada manipulación de los alimentos y la asociación de enfermedades transmitidas por alimentos (ETA), en la cual, el manipulador interviene como vehículo de transmisión.

El nitrito de sodio o potasio, al igual que los correspondientes nitratos, se utiliza de forma extensiva en el proceso de curado de muchos productos cárnicos, ya que el ion nitrito inhibe el desarrollo anaeróbico de ciertos microorganismos, especialmente del Clostridium botilinum, que ayuda a fijar el color en las carnes rojas y contribuye al desarrollo de las características organolépticas del producto (Gallignani et al. 2008). Los nitratos presentes en los productos cárnicos se reducen a la forma de nitritos, por acción de bacterias que se desarrollan en ese medio, mientras los nitritos son parcialmente reducidos a la forma de $\mathrm{NO}$, compuesto que reacciona con la mioglobina y, por esta vía, es el responsable de la coloración típica de las carnes curadas (Gallignani et al. 2008); sin embargo, en la actualidad, está bien documentado el efecto perjudicial y tóxico que ejercen los nitritos sobre la salud (Gallignani et al. 2008), ya que su consumo se asocia a enfermedades, como el cáncer, la diabetes, Parkinson y el Alzheimer (O’Flynn et al. 2014a; 2014b).

En Colombia, la fabricación de productos cárnicos, como el chorizo, no ha sido estandarizada y se monitorizan, en general, subjetivamente, y sin un control estricto de las características requeridas, para estos productos; no obstante, si se mantienen las condiciones de higiene y las propiedades intrínsecas de los alimentos, los embutidos tradicionales podrían ser considerados productos seguros (Romero et al. 2013).

Debido a que la información científica sobre el chorizo colombiano es escasa y considerando el interés social y comercial de la caracterización de productos típicos y tradicionales en la región Caribe, el objetivo de este estudio fue determinar las características microbiológicas, fisicoquímicas, nitritos y propiedades de textura de diferentes tipos o calidades de chorizos que se consumen en Cartagena de Indias D.T. y C. (Colombia), con la finalidad de establecer diferencias y contribuir a estandarizar su calidad. 


\section{MATERIALES Y MÉTODOS}

Muestras. En el estudio, se llevó a cabo un muestreo aleatorio dirigido. Se recolectaron 30 muestras al azar de diferentes chorizos de cerdo tipo coctel, comercialización en Supermercados, en Mercado de Bazurto y en venta callejera, ubicados en la ciudad de Cartagena de Indias, D.T. y C. (Colombia), tomando diez muestras de cada procedencia. Las muestras, se empacaron en bolsas de polietileno de primer uso, debidamente rotuladas, para luego ser refrigeradas.

Análisis microbiológicos, fisicoquímicos y determinación de nitritos. El contenido de nitrito fue determinado por métodos 976.14 y 973.31 de la A.O.A.C. (2003). De acuerdo a lo indicado en la Norma Técnica Colombiana 1325 de 2008, se determinó el contenido de humedad (NTC 1663, 2009), materia grasa (NTC 1662, 2008), proteína (NTC 1556, 2008), almidón (NTC 4566, 2009), recuento total aerobios (NTC 4519, 2009), coliformes totales y fecales, utilizado medio VRBL+MUG (NTC 4458, 2007), Staphylococcus aureus (NTC 4779, 2007), esporas del Clostridium spp. (NTC 4834, 2000) y Salmonella spp. (NTC 4574, 2007). Para la detección de Salmonella spp., se usó agua peptonada, como medio de pre-enriquecimiento no selectivo; Rappaport, como primer medio de enriquecimiento selectivo y, Agar XLD, como medio sólido selectivo en placa, para aislamiento.

Análisis del perfil de textura (TPA). Se utilizó un texturómetro Marca Shimadzu y se realizó la determinación del perfil de textura, por medio del Rheometer software. Para esto, se cortaron rodajas de chorizo, de aproximadamente $1,5 \mathrm{~cm}$ de grosor y se dejaron reposar por una hora, a temperatura ambiente, dentro de una bolsa de polietileno, para evitar la pérdida de humedad (Granados et al. 2013; Isaza et al. 2010). El método de TPA fue realizado mediante la aplicación de una fuerza de compresión, dos veces sucesivas en las muestras, con el fin de simular la masticación humana, obteniendo la curva fuerza/tiempo y calculados los parámetros: cohesividad, dureza, gomosidad y elasticidad.

Análisis de datos. Esta investigación empleó un diseño aleatorio completamente al azar. Las determinaciones se efectuaron por triplicado y los resultados expresados, como la media, con su desviación estándar. Se utilizó el programa PHARM/PCS versión 4, para calcular la media y la desviación estándar de los resultados en los análisis efectuados. Cuando se detectaron diferencias significativas entre las medias fueron comparadas, utilizando la prueba de diferencia mínima significativa. Se fijó el nivel de significancia a p<0,05.

El conteo de microorganismos fue en Log de Unidades Formadoras de Colonia por gramo (UFC/g), a excepción de la Salmonella, que se expresó en Salmonella/25g. Los resultados, se muestran como promedios de los datos obtenidos para cada sitio de muestreo.

\section{RESULTADOS Y DISCUSIÓN}

Análisis microbiológico. En la tabla 1, se pueden observar los resultados de recuento total de aerobios, coliformes totales y fecales, S. aureus, Clostridium spp. y Salmonella, que se realizaron a los chorizos. Según esta, los chorizos de Supermercado son los únicos que se encuentran dentro de los rangos estipulados por la NTC 1325 (2008), para índice máximo permisible, para nivel de buena calidad, en productos cárnicos procesados. Los malos resultados de los demás chorizos son valores, probablemente, relacionados, tanto con las malas prácticas higiénicas como con el uso de materias primas empleadas, para abaratar costos de producción, con altos niveles de contaminación microbiana.

En trabajos anteriores, Bayona (2012) realizó un estudio descriptivo de prevalencia, en donde se evaluó la frecuencia de ciertos microorganismos en los manipuladores y alimentos vendidos, en una zona del norte de Bogotá, referente a la carga de enteroparásitos y Salmonella spp. La población evaluada correspondió a 60 manipuladores de alimentos distribuidos, así: 40, de venta establecida y 20 , de venta ambulatoria. Este autor realizó ensayos microbiológicos en muestras de heces, de frotis de manos y de alimentos, en la búsqueda de enteroparásitos y Salmonella spp. Dentro de sus resultados, se destaca que el $25 \%$ de los alimentos ambulantes y el 7,5\% de los alimentos de venta establecida, entre ellas chorizo frito y pincho de carne, fueron positivos

Tabla 1. Características microbiológicas de chorizos analizados $(n=10)$.

\begin{tabular}{|c|c|c|c|c|c|c|}
\hline $\begin{array}{c}\text { Lugar } \\
\text { muestreo }\end{array}$ & $\begin{array}{c}\text { Aerobios } \\
\text { (UFC/g) }\end{array}$ & $\begin{array}{c}\text { Coliformes } \\
\text { totales (UFC/g) }\end{array}$ & $\begin{array}{c}\text { Coliformes } \\
\text { fecales (UFC/g) }\end{array}$ & $\begin{array}{c}\text { S. aureus } \\
\text { (UFC/g) }\end{array}$ & $\begin{array}{c}\text { Esporas } \\
\text { Clostridium spp. } \\
\text { (UFC/g) }\end{array}$ & Salmonella/25g \\
\hline Mercado de Bazurto & 12 & 11 & 5 & 2 & 0 & 0 \\
\hline Venta callejera & 16 & 15 & 10 & 5 & 0 & 0 \\
\hline Supermercado & 5 & 0 & 0 & 0 & 0 & 0 \\
\hline Permitido & 5 & 2 & 2 & $<2$ & 0 & 0 \\
\hline
\end{tabular}


para Salmonella spp. Este mismo autor resalta que la presencia de estos contaminantes microbiológicos en alimentos de venta callejera representa un aspecto de importancia en salud pública, lo que implica fomentar una cultura de higiene, en los manipuladores de alimentos.

Análisis fisicoquímicos. La tabla 2 refleja la composición de chorizos comercializados, en la ciudad de Cartagena. Las mayores diferencias estadísticas, se observaron en los contenidos de proteínas y de almidón $(\mathrm{p}<0,05)$. En la tabla 2 , se puede observar cómo el chorizo comercializado en supermercado presentó mayor contenido de proteína y menor de carbohidrato y almidón, que los obtenidos en las ventas callejeras y el mercado de Bazurto. La variación de proteína entre un chorizo y otro, se puede deber a las diferentes cantidades de carne y de grasa utilizadas en la formulación de cada tipo de chorizo (González-Tenorio et al. 2013). Las diferencias de carbohidratos y de almidón, se deben a la cantidad de rendidor que se adiciona en la formulación para cada producto, siendo este mayor en los productos de venta libre, a diferencia de los vendidos en supermercados (González-Tenorio et al. 2013). La composición proximal de estos chorizos es muy parecida a las reportadas por otros autores en chorizo (Cengiz \& Gokoglu, 2005; Jiménez-Colmenero et al. 2010; Yang et al. 2007). Pacheco et al. (2011), en su estudio de chorizo con tocino de cerdo y chorizo con extensor graso, reportó valores de humedad entre 60 y $66 \%$, proteína entre 14 y $15 \%$, grasa entre 11 y $17 \%$ y ceniza, valores cercanos al $3 \%$. El contenido de humedad para los tres tipos de chorizos es mayor que los reportados por González-Tenorio et al. (2013), para chorizos frescos y menor en los demás componentes. Probablemente, estos valores altos en el contenido de humedad se deban a que los ingredientes cárnicos, utilizados en la elaboración de los chorizos, se caracterizan por una elevada capacidad de retención de agua (Granados et al. 2013) y por el agua considerada en la formulación.

Contenido de nitritos. La tabla 3 muestra el contenido de nitritos de chorizos comercializados, en la ciudad de Cartagena. En esta, se observan diferencias estadísticas entre los distintos tipos de chorizo, dependiendo de su procedencia (p<0,05). Según la NTC 1325 (2008), la cantidad máxima admisible de nitritos en productos procesados es de $200 \mathrm{mg} /$ $\mathrm{kg}$. Según lo anterior, solamente los chorizos comercializados en supermercado cumplen con la legislación colombiana y, los demás, están fuera del rango de permisibilidad, para consumo en Colombia. De acuerdo a la NTC 1325 (2008), los nitritos prolongan la conservación del producto y la cuantía presente en chorizos de venta callejera y Mercado Bazurto encubren la gran cantidad de almidón, utilizado como rendidor y prolongan la vida útil de producto, sin importar que sobrepasen los contenidos permisibles (NTC 1325, 2008).

Sinnúmero de alimentos cárnicos procesados, como los chorizos, poseen elevados niveles de nitritos y algunos países han avanzado en el desarrollo programas para reducir, significativamente, el contenido de estos en los alimentos procesados y el fomento de una disminución en su uso indiscriminado (Doyle \& Glass, 2010; O'Flynn et al. 2014a; 2014b; Smith-Spangler et al. 2010). La reducción de estos es un problema creciente para los productores de carne, bajo la presión de los minoristas y los consumidores de productos más naturales (O'Flynn et al. 2014a), ya que su uso sin control, como conservante alimentario, se asocia a enfermedades, como el cáncer, la diabetes, Parkinson, el Alzheimer, la inhibición del transporte de oxígeno, la irritación del sistema digestivo, daños en la sangre y vasos sanguíneos, entre otros efectos negativos (O'Flynn et al. 2014a; 2014b).

Análisis de perfil de textura. En la tabla 4, se muestran los resultados obtenidos en el análisis de perfil de textura en los distintos tipos de chorizos analizados. En la textura del chorizo debe influir, decisivamente, la materia prima cárnica, la eventual presencia de almidones o proteínas no cárnicas, el diámetro del embutido y el grado de secado. El papel de la textura en la calidad de los chorizos no es del todo claro y depende de la variedad de chorizo de que se trate (González-Tenorio et al. 2013). Se observaron diferencias estadísticas en todas las características texturales entre un chorizo y otro $(p<0,05)$.

La mayor dureza la presentaron los chorizos de supermercado, debido, posiblemente, a fuerzas estructurales en la matriz

Tabla 2. Composición proximal (\%) de chorizos analizados $(n=10)$.

\begin{tabular}{|c|c|c|c|c|}
\hline Componente & Mercado de Bazurto & Venta callejera & Supermercado & Permitido Estándar \\
\hline Humedad & $66,2 \pm 4,90^{\mathrm{a}}$ & $69 \pm 5,07^{\mathrm{a}}$ & $65 \pm 4,34^{\mathrm{a}}$ & Máximo 62 \\
\hline Proteínas & $11,20 \pm 2,78^{\mathrm{a}}$ & $7,23 \pm 3,86^{\mathrm{b}}$ & $13,97 \pm 3,25^{\mathrm{c}}$ & Mínimo $10^{\mathrm{c}}$ \\
\hline Grasas & $14,2 \pm 1,45^{\mathrm{a}}$ & $14,6 \pm 1,67^{\mathrm{a}}$ & $13,8 \pm 2,56^{\mathrm{a}}$ & Máximo 28 \\
\hline Almidón & $4,21 \pm 0,24^{\mathrm{b}}$ & $10,23 \pm 0,45^{\mathrm{b}}$ & $1,34 \pm 0,57^{\mathrm{c}}$ & Máximo 10 \\
\hline
\end{tabular}

Letras diferentes significan diferencias significativas $(p<0,05)$. 
Tabla 3. Contenido de nitritos de chorizos $(n=10)$.

\begin{tabular}{|c|c|}
\hline Lugar muestreo & Nitritos $\mathrm{mg} / \mathrm{kg}$ \\
\hline Mercado de Bazurto & $220,78 \pm 2,09^{\mathrm{a}}$ \\
\hline Venta Callejera & $330,33 \pm 4,67^{\mathrm{b}}$ \\
\hline Supermercado & $20,35 \pm 1,089$ \\
\hline Permitido & $200^{\mathrm{c}}$ \\
\hline
\end{tabular}

Letras diferentes significan diferencias significativas $(p<0,05)$.

cárnica, por la retención de agua en los extensores durante el tratamiento térmico, lo que ayudó, probablemente, a la textura del extensor y, por ende, la ligazón y la distribución de los gránulos de carne en la matriz final (Pacheco et al. 2011). Los valores de cohesividad son similares a los reportados por González-Tenorio et al. (2013), en su estudio de caracterización de propiedades químicas y fisicoquímicas de chorizos comercializados, en el centro de México.

Existen variaciones notables en las características de cada chorizo, teniendo en cuenta su procedencia. Los chorizos de supermercado fueron los de menor recuento microbiano y los únicos que cumplían con la legislación colombiana, lo que hace pensar que los chorizos que presentaron mayor recuento, como los procedentes de la central de abasto y las ventas ambulantes, están siendo elaborados bajo precarias condiciones de salubridad. La regulación de las condiciones técnico-sanitarias de comercialización del chorizo, se debería mejorar en la ciudad de Cartagena de Indias (Colombia), ya que los únicos chorizos que reunieron todas las características permitidas por la ley fueron los comercializados en supermercados, lo cual, es preocupante, ya que las ventas en la central de abasto y ambulantes tienen una alta demanda.

Conflictos de intereses: El manuscrito fue preparado y revisado con la participación de todos los autores, quienes declaramos que no existe conflicto de intereses que ponga en riesgo la validez de los resultados presentados.

Tabla 4. Perfil de textura de chorizos analizados $(n=10)$.

\begin{tabular}{|c|c|c|c|}
\hline Atributo & Mercado de Bazurto & Venta Callejera & Supermercado \\
\hline Dureza(N) & $6,78 \pm 2,70^{\mathrm{a}}$ & $4,2 \pm 1,40^{\mathrm{b}}$ & $8,62 \pm 3,60^{\mathrm{c}}$ \\
\hline Adhesividad(N) & $-0,56 \pm 0,07^{\mathrm{a}}$ & $-0,43 \pm 0,06^{\mathrm{b}}$ & $-0,68 \pm 0,02^{\mathrm{c}}$ \\
\hline Cohesividad & $0,64 \pm 0,09^{\mathrm{a}}$ & $0,53 \pm 0,047^{\mathrm{b}}$ & $0,72 \pm 0,1^{\mathrm{c}}$ \\
\hline Elasticidad & $0,45 \pm 0,01^{\mathrm{a}}$ & $0,35 \pm 0,08^{\mathrm{b}}$ & $0,61 \pm 0,05^{\mathrm{c}}$ \\
\hline Masticabilidad(N) & $4,33 \pm 0,02^{\mathrm{a}}$ & $2,22 \pm 0,5^{\mathrm{b}}$ & $6,2 \pm 0,04^{\mathrm{c}}$ \\
\hline
\end{tabular}

Letras diferentes significan diferencias significativas $(p<0,05)$.

\section{BIBLIOGRAFÍA}

1. A.O.A.C. 2003. Official Methods of Analysis of the AOAC International. 18th ed. Horowitz, W.; Latimer Jr., G.W. (eds) Association of Analytical Chemists. Maryland (United States). 780p.

2. AMBROSIADIS, J.; SOULTOS, N.; ABRAHIM, A.; BLOUKAS, J.G. 2004. Physicochemical, microbiological and sensory attributes for the characterization of Greek traditional sausages. Meat Sci. 66(2):279-87.
3. BAYONA, M.A. 2012. Prevalencia de Salmonella y enteroparásitos en alimentos y manipuladores de alimentos de ventas ambulantes y restaurantes en un sector del norte de Bogotá, Colombia. Rev. U.D.C.A Act. \& Div. Cient. 15(2):267-274.

4. CENGIZ, E.; GOKOGLU, N. 2005. Changes in energy and cholesterol contents of frankfurter-type sausages with fat reduction and fat replacer addition. Food Chem. 91(3):443-447.

5. DOYLE, M.E.; GLASS, K.A. 2010. Sodium Reduction and Its Effect on Food Safety, Food Quality, and Hu- 
man Health. Comprehensive Rev. Food Sci. Food Safety. 9(1):44-56.

6. FERNÁNDEZ-LÓPEZ, J.; SENDRA, E.; SgAYAS-BARBERÁ, E.; NAVARRO, C.; PÉREZ-ÁLVAREZ, J.A. 2008. Physico-chemical and microbiological profiles of "salchichón" (Spanish dry-fermented sausage) enriched with orange fiber. Meat Sci. 80(2):410-417.

7. FOOD AND AGRICULTURE ORGANIZATION OF THE UNITED NATIONS -FAO-. 2006. Chorizo. Disponible desde Internet en http://www.fao.org/inpho_archive/content/documents/vlibrary/ae620s/pprocesados/carn4.htm. [con acceso el 02/06/2014].

8. GALLIGNANI, M.; CASTELLANOS, L.; VALERO, M.; BRUNETTO, M. 2008. Determinación de nitritos en chorizos por espectrofotometría derivativa, utilizando un sistema de análisis en flujo. Ciencia. 16(2):241250.

9. GONZÁLEZ, B.; DÍEZ, V. 2002. The effect of nitrite and starter culture on microbiological quality of "chorizo"a Spanish dry cured sausage. Meat Sci. 60(3):295298.

10. GONZÁLEZ-TENORIO, R.; CARO, I.; SOTO, S.; RODRÍGUEZ, B.R.; MATEO, J. 2012. Características microbiológicas de cuatro tipos de chorizo comercializados en el Estado de Hidalgo. Nacameh. 6(2):25-32.

11. GONZALEZ-TENORIO, R.; TOTOSAUS, A.; CARO, I.; MATEO, J. 2013. Caracterización de Propiedades Químicas y Fisicoquímicas de Chorizos Comercializados en la Zona Centro de México. Información Tecnológica. 24(2):3-14.

12. GRANADOS, C.; GUZMÁN, L.E.; ACEVEDO, D. 2013. Análisis proximal, sensorial y de textura de salchichas elaboradas con subproductos de la industria procesadora de atún (Scombridae thunnus). Inf. Tecnol. 24(6):29-34.

13. GUERRERO, L.; GUÀRDIA, M.D.; XICOLA, J.; VERBEKE, W.; VANHONACKER, F.; ZAKOWSKA-BIEMANS, S.; HERSLETH, M. 2009. Consumer-driven definition of traditional food products and innovation in traditional foods. A qualitative cross-cultural study. Appetite. 52(2):34554.

14. ISAZA, J.M.; LONDOÑO, L.M.; RESTREPO, D.; CORTÉS, M.; SUÁREZ, H. 2010. Producción y propiedades funcionales de plasma bovino hidratado en embutido tipo salchichón. Rev. Col. Cienc. Pec. 23(2):199-206.

15. JIMÉNEZ-COLMENERO, F.; HERRERO, A.; PINTADO, T.; SOLAS, M.T.; RUIZ-CAPILLAS, C. 2010. Influence of emulsified olive oil stabilizing system used for pork backfat replacement in frankfurters. Food Res. Int. 43(8):2068-2076.

16. NTC 1325. 2008. Instituto Colombiano de Normas Técnicas y Certificación (Colombia). Industrias alimentarias. Productos carnicos procesados no enlatados. Quinta Actualización, Bogotá.

17. NTC 1556. 2008. Instituto Colombiano de Normas Técnicas y Certificación (Colombia). Carne y productos cárnicos. Método para determinar el contenido de nitrógeno (Método de referencia y de rutina). Segunda Actualización, Bogotá.

18. NTC 1662. 2008. Instituto Colombiano de Normas Técnicas y Certificación (Colombia). Carne y productos cárnicos. Métodos de determinación del contenido de grasa total. Método de referencia y método de rutina. Primera Actualización, Bogotá.

19. NTC 1663. 2009. Instituto Colombiano de Normas Técnicas y Certificación (Colombia). Carne y productos cárnicos. Métodos de determinación del contenido de humedad. Método de referencia y método de rutina. Segunda Actualización, Bogotá.

20. NTC 4458. 2007. Instituto Colombiano de Normas Técnicas y Certificación (Colombia). Microbiología de alimentos y de alimentos para animales. Método horizontal para el recuento de coliformes o Escherichia coli o ambos. Técnica de recuento de colonias utilizando medios fluorogénicos o cromogénicos. Primera Actualización, Bogotá.

21. NTC 4519. 2009. Instituto Colombiano de Normas Técnicas y Certificación (Colombia). Microbiología de los alimentos para consumo humano y animal. Método horizontal para el recuento de microorganismos. Técnica de recuento de colonias a $30^{\circ} \mathrm{C}$. Primera Actualización, Bogotá.

22. NTC 4566. 2009. Instituto Colombiano de Normas Técnicas y Certificación (Colombia). Productos cárnicos. Determinación del contenido de almidón (métodos de referencia). Primera Actualización, Bogotá.

23. NTC 4574. 2007. Instituto Colombiano de Normas Técnicas y Certificación (Colombia). Microbiología de al- 
imentos y de alimentos para animales. Método horizontal para la detección de Salmonella spp. Primera Actualización, Bogotá.

24. NTC 4779. 2007. Instituto Colombiano de Normas Técnicas y Certificación (Colombia). Microbiología de alimentos y alimentos para animales. Método horizontal para el recuento de estafilococos coagulasa positiva (Staphylococcus aureus y otras especies). Primera Actualización, Bogotá.

25. NTC 4834. 2000. Instituto Colombiano de Normas Técnicas y Certificación (Colombia). Microbiología de alimentos y alimentos para animales. Método horizontal para el recuento de Clostridium sulfito reductores e identificación de Clostridium perfringens - técnicas de recuento de colonias. Primera Actualización, Bogotá.

26. O'FLYNN, C.C.; CRUZ-ROMERO, M.C.; TROY, D.J.; MULLEN, A.M.; KERRY, J.P. 2014a. The application of high-pressure treatment in the reduction of phosphate levels in breakfast sausages. Meat Sci. 96(1):633-639.

27. O'FLYNN, C.C.; CRUZ-ROMERO, M.C.; TROY, D.; MULLEN, A.M.; KERRY, J.P. 2014b. The application of high-pressure treatment in the reduction of salt levels in reduced-phosphate breakfast sausages. Meat Sci. 96(3):1266-1274.
28. PACHECO, W.; RESTREPO, D.; LÓPEZ, J. 2011. Evaluación de un Extensor Graso sobre las Propiedades de Calidad del Chorizo Tipo Antioqueño. Rev. Fac. Nal Agron., Medellín. 64(2):6265-6276.

29. ROMERO, M.C.; ROMERO, A.M.; DOVAL, M.M.; JUDIS, A. 2013. Nutritional value and fatty acid composition of some traditional Argentinean meat sausages. Food Sci. Techn. 33(1):161-166.

30. SMITH-SPANGLER, C.M.; JUUSOLA, J.L.; ENNS, E.A.; OWENS, D.K.; GARBER, A.M. 2010. Population strategies to decrease sodium intake and the burden of cardiovascular disease: a cost-effectiveness analysis. Ann. Internal Med. 152(8):481-487.

31. WANG, X.H.; REN, H.Y.; LIU, D.Y.; ZHU, W.Y.; WANG, W. 2013. Effects of inoculating Lactobacillus sakei starter cultures on the microbiological quality and nitrite depletion of Chinese fermented sausages. Food Control. 32(2):591-596.

32. YANG, H.S.; CHOI, S.G.; JEON, J.T.; PARK, G.B.; JOO, S.T. 2007. Textural and sensory properties of low fat pork sausages with added hydrated oatmeal and tofu as texture-modifying agents. Meat Sci. 75(2):283289.

Recibido: Agosto 9 de 2014

Aceptado: Enero 14 de 2015

Cómo citar:

Tirado, D.; Acevedo, D.; Montero, P. 2015. Calidad microbiológica, fisicoquímica, determinación de nitritos y textura de chorizos comercializados en Cartagena (Colombia). Rev. U.D.C.A Act. \& Div. Cient. 18(1): 189-195. 